TITLE:

\title{
Fluid motions induced by horizontally heterogeneous Joule heating in the Earth's inner core
}

\author{
$\operatorname{AUTHOR}(S)$ :
}

Takehiro, Shin-Ichi

\section{CITATION:}

Takehiro, Shin-Ichi. Fluid motions induced by horizontally heterogeneous Joule heating in the Earth's inner core. Physics of the Earth and Planetary Interiors 2011, 184(3-4): 134-142

\section{ISSUE DATE:}

2011-02

URL:

http://hdl.handle.net/2433/197332

\section{RIGHT:}

(c) 2010 Elsevier B.V. NOTICE: this is the author's version of a work that was accepted for publication in Physics of the Earth and Planetary Interiors. Changes resulting from the publishing process, such as peer review, editing, corrections, structural formatting, and other quality control mechanisms may not be reflected in this document. Changes may have been made to this work since it was submitted for publication. A definitive version was subsequently published in Physics of the Earth and Planetary Interiors, 184(3-4), 2011, doi:10.1016/j.pepi.2010.11.002; This is not the published version. Please cite only the published version.; この論文は出版社版でありません。引用の際には出版社版をご確認ご 利用ください。 


\title{
Fluid motions induced by horizontally heterogeneous Joule heating in the Earth's inner core
}

\author{
Shin-ichi Takehiro ${ }^{* a}$ \\ ${ }^{a}$ Research Institute for Mathematical Sciences, Kyoto University, Sakyou-ku, Kyoto, \\ 606-8502 Japan
}

\begin{abstract}
Horizontally heterogeneous Joule heating is proposed as a new driving source for fluid motions in the Earth's inner core. The magnetic field imposed at the inner core boundary (ICB) penetrates into the inner core through diffusion and generates Joule heating. When the heating distribution is horizontally heterogeneous, it produces torque by means of the buoyancy force, thereby inducing fluid motions in the inner core. The expression of fluid flows induced by arbitrary magnetic field distributions at ICB is obtained analytically.

Using the estimated values of the physical parameters of the inner core, the amplitude of the stress field associated with the flows induced by this mechanism is expected to be greater than or approximately the same as that of the models considered thus far, and is sufficiently large for large scale deformation of the inner core. The flow field by this mechanism is also accompanied by a weak stress field layer near the ICB. The thickness of this boundary layer is comparable to the depth of the weak anisotropy region observed near the ICB.

The model presented herein suggests that interactions of the flow and magnetic fields through Joule heating may occur between the inner and outer cores. The flow field induced by Joule heating generates mass exchange through the ICB, causing absorption and release of latent heat and light elements. This process affects the flow field and the dynamo action in the outer core and possibly
\end{abstract}

*Corresponding author. Email:takepiro@gfd-dennou.org, Tel:+81-75-753-7260, Fax:+8175-753-7272. 
reflects on the distribution of the magnetic field. The variation of the magnetic field penetrates the ICB again and modifies the distribution of Joule heating and the resultant flow field in the inner core.

Key words: Inner core flows, Joule heating, Seismic anisotropy, Dynamo, Interaction between the inner and outer cores

\section{Introduction}

Recent seismological observations have indicated anisotropy of seismic wave velocities in the inner core, and the existence of this anisotropy has come to be widely accepted (e.g., Poupinet et al., 1983; Morelli et al., 1986; Souriau, 2007, for recent reviews). The finding that the wave velocity in the polar direction is $3 \%$ faster than that in the equatorial plane is thought to be explained by the alignment of the preferred orientation of hexagonal close packed (h.c.p.) iron crystals (e.g., Jeanloz, 1990). Whereas anisotropic crystal growth under the circumstance of the inner core is proposed as the alignment mechanism (e.g., Karato, 1993; Bergman, 1997), several theories attribute the crystal alignment to fluid motions in the inner core as described below.

Jeanloz and Wenk (1988) reported that the amount of radioactive elements in the inner core would be sufficient to drive thermal convection and suggested that convective fluid motions with a horizontal structure of the spherical harmonic degree 1 component could align the preferred orientation of the iron crystal. However, according to recent calculations of the thermal history, it is doubtful that the inner core is thermally unstable, because radioactive elements captured inside the inner core are insufficient and the cooling rate of the core is too slow (e.g., Yukutake, 1998; Buffett, 2009). Another problem with this model is that there is no reason for the axis of the convection cell to be directed poleward.

Yoshida et al. (1996) and Sumita and Yoshida (2003) suggested that the horizontal heterogeneous growth of the inner core induces axisymmetric fluid flows directed parallel to the rotation axis. They believed that efficient cooling at low-latitudes due to the columnar convection in the outer core would cause 
heterogeneous growth with the $Y_{2}^{0}$ horizontal pattern of the spherical harmonics. Then, they showed that the inner core deforms isostatically in order to maintain its spherical shape and that fluid flows from the equatorial region to the polar regions are induced. Moreover, they theoretically calculated the crystal alignment caused by this flow and demonstrated that this model can explain the seismic anisotropy of the inner core. However, the disadvantage of their model is its small amplitude of velocity. Since the order of the velocity is comparable to the growth rate of the inner core, it will take a geologically long time for the generation of the anisotropy.

In an attempt to explain why the axis of the fluid flow is directed parallel to the rotating axis, Karato (1999) considered the dynamic effect of the magnetic field at the inner core boundary (ICB) on the inner core. He reported that the normal component of the Maxwell stresses of the geomagnetic field generated in the outer core operates at the ICB and compresses the inner core, inducing sufficiently strong fluid flows and stresses to generate the seismic anisotropy. Since the distribution of the induced fluid flow in the inner core is governed by the pattern of the geomagnetic field at the ICB in this model, he also expected that the distribution of seismic anisotropy would reflect the structure of the geomagnetic field in the outer core. However, Buffett and Bloxham (2000) argued that the Maxwell stress imposed by the outer core would balance the other stresses, such as the buoyancy, and they questioned whether strong fluid flows in the inner core could be induced through this mechanism.

Buffett and Wenk (2001) considered a shear component of the Maxwell stress rather than a normal component. They calculated the seismic anisotropy associated with the induced shear flows and showed that the preferred orientation of the aligned crystals is qualitatively consistent with the seismic observations. However, the strength of the Maxwell stresses of this model appear to be insufficient compared with the necessary amplitude for the generation of the anisotropy, and the distribution of the stress fields strongly depends on the unrecognized morphology of the geomagnetic field around the ICB.

Buffett (2009) examined the effects of the centrifugal force on thermal con- 
vection in the inner core, and showed that the structure of spherical harmonic degree 1 in which the axis of upward motions align with the rotation axis emerges at the critical state. Then he argued that such a coherent structure might appear and bring the anisotropic crystals alignment just before termination of thermal convection, which had been possibly vigorous at the initial stage of the thermal history of the inner core. However, it is not clear whether the amplitude and duration of velocity and stress fields associated with nearly critical thermal convection were sufficient for the crystal alignment.

In this manner, geophysicists have expressed interest in the fluid motions in the inner core as a possible origin of the seismic anisotropy. However, none of the models proposed thus far have been adequate, and each of these models has its own advantages and disadvantages. Nevertheless, the concepts proposed by Karato (1999) and Buffett and Bloxham (2000) are interesting because they demonstrated that the inner core could be affected by the magnetic field in the outer core. They considered the dynamic effects through the Maxwell stress or the Lorentz force of the geomagnetic field. However, the effects of the magnetic field are not necessarily limited to the dynamic effects. The thermal effect through the Joule heating might act on the inner core. Since the amplitude of Joule heating in the inner core is considered to be small compared with other thermal factors, such as global secular cooling of the inner core, the effect of Joule heating is usually neglected, for example, in discussions of the occurrence of thermal convection or the thermal history of the inner core. Horizontal variation of Joule heating, however small, can induce a horizontal temperature gradient and cause torque associated with the buoyancy force, which drives horizontal convective fluid motions.

The present study investigates fluid motions in the inner core induced by horizontally heterogeneous Joule heating of the magnetic field imposed by the outer core. The strength of stresses associated with induced flows is estimated. The model formulation is presented in Section 2, and steady solutions are obtained analytically in Section 3. Geophysical applications are discussed in Section 4. Section 5 summarizes the results. 


\section{Model}

As a model of the inner core, let us consider a sphere of radius $a$ filled with a Boussinesq fluid. As a basic state, we assume the magnetic field in the inner core, $\boldsymbol{B}_{\mathbf{0}}(\boldsymbol{r})$, imposed through the ICB from the outer core and spherically symmetric temperature field, $T_{0}(r)$, induced by the secular cooling process, where $r$ is the radius from the center of the sphere, and $\boldsymbol{r}$ denotes the position vector.

The magnetic field of the basic state is determined by a given distribution field at the ICB:

$$
\boldsymbol{B}_{\mathbf{0}}=\boldsymbol{B}_{\boldsymbol{s}}(\theta, \phi) \quad \text { at } \quad r=a,
$$

and the steady induction equation with no fluid motion and the solenoidal condition of the field:

$$
\boldsymbol{\nabla} \times \boldsymbol{\nabla} \times \boldsymbol{B}_{\mathbf{0}}=0, \quad \boldsymbol{\nabla} \cdot \boldsymbol{B}_{\mathbf{0}}=0,
$$

where $\theta$ and $\phi$ are the colatitude and azimuth, respectively, in the spherical coordinate. The solutions of Eqs. (1) and (2) are expressed by introducing toroidal and poloidal potentials $\mathcal{T}(\boldsymbol{r})$ and $\mathcal{P}(\boldsymbol{r})$ as follows:

$$
\begin{aligned}
& \mathcal{T}(r, \theta, \phi)=\sum_{n, m} \tilde{\mathcal{T}}_{n m} r^{n} Y_{n}^{m}(\theta, \phi), \\
& \mathcal{P}(r, \theta, \phi)=\sum_{n, m} \tilde{\mathcal{P}}_{n m} r^{n} Y_{n}^{m}(\theta, \phi),
\end{aligned}
$$

where $\boldsymbol{B}=\boldsymbol{\nabla} \times(\mathcal{T} \boldsymbol{r})+\boldsymbol{\nabla} \times \boldsymbol{\nabla} \times(\mathcal{P} \boldsymbol{r}) . Y_{n}^{m}(r, \theta, \phi)$ is a spherical harmonics function of degree $n$ and order $m$. The amplitude of each spherical harmonics component $\tilde{\mathcal{T}}_{n m}$ and $\tilde{\mathcal{P}}_{n m}$ is determined by the magnetic field distribution at the ICB, $\boldsymbol{B}_{\boldsymbol{s}}(\theta, \phi)$. The Joule heating $Q_{J}$ associated with the magnetic field is as follows:

$$
Q_{J} \equiv \frac{\left|\boldsymbol{J}_{\mathbf{0}}\right|^{2}}{\sigma}=\frac{\left|\boldsymbol{\nabla} \times \boldsymbol{B}_{\mathbf{0}}\right|^{2}}{\mu^{2} \sigma}
$$

where $\boldsymbol{J}_{\mathbf{0}}$ is the electric current field of the basic state, and $\sigma$ and $\mu$ are the electrical conductivity and the magnetic permeability, respectively. Note that the poloidal fields do not accompany electric currents. The electric current field is expressed by the potentials as $\boldsymbol{J}_{\mathbf{0}}=\boldsymbol{\nabla} \times \boldsymbol{\nabla} \times(\mathcal{T} \boldsymbol{r})-\boldsymbol{\nabla} \times\left(\nabla^{2} \mathcal{P} \boldsymbol{r}\right)$. The second term of the right-hand side vanishes because the solution (3) satisfies $\nabla^{2} \mathcal{P}=0$. 
Therefore, it is sufficient to consider the toroidal magnetic field only, and the poloidal fields are not relevant to the present problem.

The equations for disturbances with respect to this basic state are as follows:

$$
\begin{aligned}
& \frac{\partial \boldsymbol{v}}{\partial t}+(\boldsymbol{v} \cdot \boldsymbol{\nabla}) \boldsymbol{v}+2 \boldsymbol{\Omega} \times \boldsymbol{v}=-\frac{1}{\rho_{0}} \boldsymbol{\nabla}+\frac{1}{\rho_{0}} \boldsymbol{J} \times \boldsymbol{B}_{0}+\frac{1}{\rho_{0}} \boldsymbol{J}_{0} \times \boldsymbol{B}+\alpha T \boldsymbol{g}+\nu \boldsymbol{\nabla}^{2}(\boldsymbol{\boldsymbol { r }}) \\
& \frac{\partial T}{\partial t}+(\boldsymbol{v} \cdot \boldsymbol{\nabla}) T+v_{r} \frac{d T_{0}}{d r}=\kappa \nabla^{2} T+\frac{Q_{J}}{\rho_{0} C_{p}} \\
& \frac{\partial \boldsymbol{B}}{\partial t}=\boldsymbol{\nabla} \times\left(\boldsymbol{v} \times \boldsymbol{B}_{0}\right)+\lambda \boldsymbol{\nabla}^{2} \boldsymbol{B} \\
& \boldsymbol{\nabla} \cdot \boldsymbol{v}=0, \quad \boldsymbol{\nabla} \cdot \boldsymbol{B}=0
\end{aligned}
$$

Here, $\boldsymbol{v}, v_{r}, T$, and $\boldsymbol{B}$ are the velocity, radial component of velocity, temperature, and magnetic field disturbances induced by Joule heating, respectively. In addition, $\boldsymbol{\Omega}$ is the rotation rate of the system, $\alpha$ is the thermal expansion coefficient, $\nu$ is the kinematic viscosity, $\kappa$ is the thermal diffusivity, $\rho_{0}$ is the average density of the Boussinesq fluid, $C_{p}$ is the specific heat capacity, and $\lambda$ is the magnetic diffusivity. Here, $\boldsymbol{g}$ is assumed to be the spherically symmetric self-gravitational acceleration of the sphere and is expressed as $\boldsymbol{g}=\left(g_{0} / a\right) \boldsymbol{r}$, where $g_{0}$ is the acceleration due to gravity at the ICB. Since we are interested in fluid flows induced by Joule heating only, the Lorentz force of the basic magnetic field $\boldsymbol{J}_{0} \times \boldsymbol{B}_{0} / \rho_{0}$, which is examined in Buffett and Bloxham (2000), is removed from Eq. (5).

Note that in the framework of the Boussinesq approximation, the basic temperature gradient $d T_{0}(r) / d r$ expresses the difference from the adiabatic temperature gradient (potential temperature gradient). Therefore, $d T_{0}(r) / d r=0$ means neutral stratification, and $d T_{0}(r) / d r>0$ and $d T_{0}(r) / d r<0$ expresses stable and unstable stratification, respectively. It is still under debate whether the inner core of the Earth is thermally stable or unstable: for example, it may depend on the estimation of thermal conductivity of iron under the high pressure-temperature environment (e.g. Buffett, 2009). In the following, we consider the stably stratified case $d T_{0}(r) / d r>0$, for the first step of the study.

Before non-dimensionalizing the governing equations, let us discuss about the dominant balances in the equations of motion and temperature. One of 
the possibilities is that the thermal conduction term balances with the Joule heating term in the temperature equation and the induced temperature disturbance drives the fluid motion through the buoyancy force in the equation of motion. However, as you see below, this hypothesis fails because the amplitude of advection of the temperature exceeds that of the thermal conduction term. By using typical estimated values of the permeability and electrical conductivity, $\mu=4 \pi \times 10^{-7} \mathrm{H} / \mathrm{m}$, and $\sigma=2 \times 10^{5} \mathrm{Sm}^{-1}$ (Stacey and Davis, 2008), the radius of the inner core, $a=10^{6} \mathrm{~m}$, and the magnetic field strength at the ICB, $\left|\boldsymbol{B}_{0}\right|=10^{-2} \mathrm{~T}$, the amplitude of the Joule heating $\left|Q_{J}\right|$ is estimated as,

$$
\left|Q_{J}\right|=\frac{\left|\boldsymbol{B}_{0}\right|^{2}}{\mu^{2} \sigma a^{2}} \sim 3 \times 10^{-10} \mathrm{~W} / \mathrm{m}^{3} .
$$

When the thermal conduction term is balanced with this Joule heating, the magnitude of temperature disturbance $\Delta T_{d}^{\prime}$ becomes,

$$
\Delta T_{d}^{\prime} \sim \frac{\left|Q_{J}\right| a^{2}}{\rho C_{p} \kappa} \sim 10 \mathrm{~K} .
$$

where the typical values of density, specific heat capacity and thermal diffusivity, $\rho=10^{4} \mathrm{~kg} / \mathrm{m}^{3}, C_{p}=700 \mathrm{~J} / \mathrm{kg} \mathrm{K}, \kappa=5 \times 10^{-6} \mathrm{~m}^{2} / \mathrm{s}$ are adopted. The velocity amplitude $V_{d}$ induced by this temperature perturbation is calculated with the balance between the buoyancy and viscous terms as,

$$
V_{d} \sim \frac{\alpha g \Delta T_{d}^{\prime} a^{2}}{\nu} \sim 5 \times 10^{-5} \mathrm{~m} / \mathrm{s}
$$

where we use the values of viscosity, $\nu=\eta / \rho=10^{17} \mathrm{~Pa} \cdot \mathrm{s} / 10^{4}=10^{13} \mathrm{~m}^{2} / \mathrm{s}$, thermal expansion coefficient, $\alpha=1 \times 10^{-5} / \mathrm{K}$, and gravity, $g_{o}=5 \mathrm{~m} / \mathrm{s}^{2}$. Under this circumstance, the ratio of the advection and diffusion terms in the temperature equation becomes,

$$
\frac{|(\boldsymbol{v} \cdot \nabla) T|}{\left|\kappa \nabla^{2} T\right|} \sim \frac{V_{d} \Delta T_{d}^{\prime} / a}{\kappa \Delta T_{d}^{\prime} / a^{2}} \sim 10^{7}
$$

This contradicts the assumption that thermal conduction term dominates the temperature equation.

Accordingly, let us assume that the advection of temperature disturbance balances with the Joule heating. Expressing the amplitudes of the induced 
temperature disturbance and velocity, as $\Delta T_{a}^{\prime}$ and $V_{a}$, and assuming the balance between the buoyancy and viscous terms, we have,

$$
\frac{V_{a} \Delta T_{a}^{\prime}}{a} \sim \frac{\left|Q_{J}\right|}{\rho C_{p}}, \quad V_{a} \sim \frac{\alpha g \Delta T_{a}^{\prime} a^{2}}{\nu} .
$$

Then we obtain,

$$
\Delta T_{a}^{\prime} \sim\left(\frac{\nu}{\alpha g a} \frac{\left|Q_{J}\right|}{\rho C_{p}}\right)^{1] 2} \sim 3 \times 10^{-3} \mathrm{~K}, V_{a} \sim \frac{\alpha g \Delta T_{a}^{\prime} a^{2}}{\nu} \sim 1 \times 10^{-8} \mathrm{~m} / \mathrm{s} .
$$

The amplitude of temperature disturbance becomes rather small compared to that of the basic (potential) temperature, which is considered to be $\mathrm{O}(10) \mathrm{K}$ (e.g. Yukutake, 1998; Buffett and Bloxham, 2000). As a result, the advection of the basic temperature dominates the advection of temperature disturbance.

Therefore, it is expected that the advection of the basic temperature balances with the Joule heating in the equation of temperature while in the equation of motion, buoyancy and viscous terms would be in balance because of large viscosity in the inner core. That is,

$$
v_{r} \frac{d T_{0}}{d r} \sim \frac{Q_{J}}{\rho_{0} C_{p}}, \quad \alpha T \boldsymbol{g} \sim \nu \nabla^{2} \boldsymbol{v} .
$$

These relations mean that velocity field is determined by the equation of temperature, while temperature disturbance is by the equation of motion.

Based on the expected main balances discussed above, let us non-dimensionalize the governing equations. The typical magnitude of Joule heating $\left|Q_{J}\right|$ is estimated as $\left|Q_{J}\right|=B_{0}^{2} /\left(\mu^{2} \sigma a^{2}\right)$, where $B_{0}$ is a typical magnitude of the basic magnetic field at the ICB. The time scale $\tau$ is selected as $\tau=\Delta T \rho C_{p} /\left|Q_{J}\right|$, where $\Delta T$ denotes the typical value of the difference between the basic and the adiabatic temperature. Also note that $\left|Q_{J}\right| / \rho C_{p}$ is the rate of temperature increase. The length scale is the radius of the sphere $a$. The velocity scale $V$ is selected as $V=a / \tau=\left(\left|Q_{J}\right| a\right) /\left(\rho C_{p} \Delta T\right)$, which is derived from the balance between the advection of the basic temperature and Joule heating. The temperature disturbance is normalized with $(\nu V) /\left(\alpha g_{0} a^{2}\right)$ by considering the balance of the buoyancy force and the viscous force in the equation of motion. The amplitude of the magnetic field disturbance is estimated to be $\left(V a B_{0}\right) / \lambda$ based on the 
balance between the induction and the magnetic diffusion terms in the induction equation. The pressure term is scaled with $\rho_{0} \nu V / a$. The non-dimensionalized equations governing the steady state are then obtained as follows:

$$
\begin{aligned}
& R e(\boldsymbol{v} \cdot \nabla) \boldsymbol{v}+\frac{1}{E} \boldsymbol{e}_{z} \times \boldsymbol{v}=-\nabla p+M^{2}\left(\boldsymbol{J} \times \boldsymbol{B}_{0}+\boldsymbol{J}_{0} \times \boldsymbol{B}\right)+T \boldsymbol{r}+\nabla^{2} \boldsymbol{v}(9) \\
& \frac{P e}{R}(\boldsymbol{v} \cdot \nabla) T+v_{r} \frac{d T_{0}}{d r}=\frac{1}{R} \nabla^{2} T+q_{J}, \\
& 0=\nabla \times\left(\boldsymbol{v} \times \boldsymbol{B}_{0}\right)+\nabla^{2} \boldsymbol{B}, \\
& \nabla \cdot \boldsymbol{v}=0, \quad \nabla \cdot \boldsymbol{B}=0,
\end{aligned}
$$

where $q_{J}$ is the normalized Joule heating term, $R e$ is the Reynolds number, $E$ is the Ekman number, $M$ is the Hartmann number, $P e$ is the Péclet number, and $R$ is the Rayleigh number with a negative sign, which expresses the strength of the stability of the basic temperature profile.

$$
R e=\frac{V a}{\nu}, \quad E=\frac{\nu}{2 \Omega a^{2}}, \quad M=\frac{B_{0} a}{\sqrt{\rho_{0} \mu \lambda \nu}}, \quad P e=\frac{V a}{\kappa}, \quad R=\frac{\alpha g_{0} \Delta T a^{3}}{\kappa \nu} .
$$

Using the expected values of the inner core parameters, the values of the nondimensional numbers are evaluated and the magnitude of each term is compared in order to simplify the governing equations. In the inner core, the typical values of several physical properties are estimated as $a=10^{6} \mathrm{~m}, \rho=10^{4} \mathrm{~kg} / \mathrm{m}^{3}$, $C_{p}=700 \mathrm{~J} / \mathrm{kg} \mathrm{K}, \mu=4 \pi \times 10^{-7} \mathrm{H} / \mathrm{m}, \sigma=2 \times 10^{5} \mathrm{Sm}^{-1} \lambda=1.0 \mathrm{~m}^{2} \cdot \mathrm{s}^{-1}$, $\alpha=1 \times 10^{-5} / \mathrm{K}, \kappa=5 \times 10^{-6} \mathrm{~m}^{2} / \mathrm{s}, g_{o}=5 \mathrm{~m} / \mathrm{s}^{2}$, and $\Omega=7.3 \times 10^{-5}$ $\mathrm{s}^{-1}$ (Stacey and Davis, 2008). Although estimates of inner core viscosity vary from $10^{13}$ to $10^{21} \mathrm{~Pa} \mathrm{~s}$ (Sumita and Bergman, 2007), here we will assume a moderate value that is similar to that used in Karato (1999), namely, $\nu=$ $\eta / \rho=10^{17} \mathrm{~Pa} \mathrm{~s} / 10^{4}=10^{13} \mathrm{~m}^{2} / \mathrm{s}$. In addition, based on Buffett and Bloxham (2000), we assume that $\Delta T=30 \mathrm{~K}$. Although the magnitude of the toroidal magnetic field at the ICB remains unclear, we assume $B_{0}=10^{-2} \mathrm{~T}$, which is a plausible value. The values of the non-dimensional numbers are then obtained as follows:

$$
R e \sim 4 \times 10^{-18}, \quad M \sim 3 \times 10^{-2}, \quad E \sim 7 \times 10^{4}, \quad P e \sim 1, \quad R \sim 3 \times 10^{7} .
$$


Based on the values of $R e, M$, and $E$, the inertia term, the Lorentz force term, and the Coriolis term are found to be negligible compared to the viscous term. Based on the values of $P e$ and $R$, the thermal diffusion term and the advection term of temperature disturbance are assumed to be smaller than the advection term of the basic temperature. However, in this case, a thin boundary layer would form near the ICB in order to satisfy a certain boundary condition, where the effect of thermal diffusion cannot be neglected because of the small transversal length scale of the layer. Therefore, we do not neglect the thermal diffusion term.

From the scaling discussed above, we can obtain the set of equations for determining flow and temperature fields induced by Joule heating.

$$
\begin{aligned}
& 0=-\nabla p+T \boldsymbol{r}+\nabla^{2} \boldsymbol{v} \\
& v_{r} \frac{d T_{0}}{d r}=\frac{1}{R} \nabla^{2} T+q_{J} \\
& \nabla \cdot \boldsymbol{v}=0
\end{aligned}
$$

As the boundary conditions at the ICB, for simplicity, we adopt a constant normal component of the stress field, a zero shear component of the stress field, and zero temperature disturbances.

$$
\begin{aligned}
& \sigma_{r r}=-p+2 \frac{\partial v_{r}}{\partial r}=0, \\
& \sigma_{r \theta}=\frac{1}{r} \frac{\partial v_{r}}{\partial \theta}+\frac{\partial v_{\theta}}{\partial r}-\frac{v_{\theta}}{r}=0, \\
& \sigma_{r \phi}=\frac{\partial v_{\phi}}{\partial r}-\frac{v_{\phi}}{r}+\frac{1}{r \sin \theta} \frac{\partial v_{r}}{\partial \phi}=0, \\
& T=0, \quad \text { at } \quad r=1,
\end{aligned}
$$

where $v_{i}$ and $\sigma_{i j}(i, j=r, \theta, \phi)$ denote the individual components of the velocity and stress fields, respectively. These boundary conditions imply that the position of the ICB does not move, which would be realized by sufficiently fast phase change between the solid inner core and liquid outer core. 


\section{Steady analytic solutions}

In order to solve Eqs. (15) through (17), we introduce toroidal and poloidal potentials to express non-divergent velocity fields.

$$
\boldsymbol{v}=\boldsymbol{\nabla} \times(\Psi(r, \theta, \phi) \boldsymbol{r})+\nabla \times \nabla(\Phi(r, \theta, \phi) \boldsymbol{r}) .
$$

Substituting this into Eq. (15) and operating $\boldsymbol{r} \cdot \boldsymbol{\nabla} \times$ and $\boldsymbol{r} \cdot \boldsymbol{\nabla} \times \boldsymbol{\nabla} \times$, we obtain the equations for the toroidal and poloidal potentials. Expressing the equation of temperature, i.e., Eq. (16), with the potentials, we have

$$
\begin{aligned}
& \nabla^{2} L_{2} \Psi=0, \\
& \nabla^{2} L_{2} \nabla^{2} \Phi-L_{2} T=0, \\
& \frac{L_{2} \Phi}{r} \frac{d T_{0}}{d r}=\frac{1}{R} \nabla^{2} T+q_{J}
\end{aligned}
$$

where $L_{2}$ is horizontal Laplacian operator on a unit sphere with negative sign. Equation (23) indicates that the toroidal component of velocity is not induced and $\Psi \equiv 0$.

Removing the temperature term from Eqs. (24) and (25), the equation for poloidal potential is obtained as follows:

$$
\frac{L_{2} \Phi}{r} \frac{d T_{0}}{d r}-\frac{1}{R} \nabla^{2} \nabla^{2} \nabla^{2} \Phi=q_{J}
$$

Here, let us express the boundary conditions given in Eqs. (18) through (21) with the poloidal potential. Using Eq. (24), Eq. (21) is rewritten as follows:

$$
\nabla^{2} \nabla^{2} \Phi=0 \quad \text { at } \quad r=1 .
$$

The boundary condition for the normal component of the stress field is modified by removing the pressure term from Eq. (18) and the horizontal divergence from Eq. (15).

$$
\frac{\partial}{\partial r} r\left(\nabla^{2} \Phi+\frac{2 L_{2} \Phi}{r^{2}}\right)=0 \quad \text { at } \quad r=1 .
$$

By expressing the boundary condition for the shear components of the stress field given in Eqs. (19) and (20) with the poloidal potentials and organizing them into a single equation, we obtain

$$
\frac{\partial^{2} \Phi}{\partial r^{2}}-\frac{2 \Phi}{r^{2}}+\frac{L_{2} \Phi}{r^{2}}=0, \quad \text { at } \quad r=1
$$


The variables are then expanded by the spherical harmonics function series, as follows:

$\Phi=\sum_{n, m} \tilde{\Phi}_{n m}(r) Y_{n}^{m}(\theta, \phi), \quad T=\sum_{n, m} \tilde{T}_{n m}(r) Y_{n}^{m}(\theta, \phi), \quad q_{J}=\sum_{n, m} \tilde{q}_{J n m}(r) Y_{n}^{m}(\theta, \phi)$.

Substituting Eq. (30) into Eqs. (26) and (24), we have

$$
\begin{aligned}
& \frac{n(n+1) \tilde{\Phi}_{n m}}{r} \frac{d T_{0}}{d r}-\frac{1}{R} D_{n} D_{n} D_{n} \tilde{\Phi}_{n m}=\tilde{q}_{J n m}, \\
& \tilde{T}_{n m}=D_{n} D_{n} \tilde{\Phi}_{n m},
\end{aligned}
$$

where

$$
D_{n}=\frac{1}{r^{2}} \frac{d}{d r} r^{2} \frac{d}{d r}-\frac{n(n+1)}{r^{2}} .
$$

The boundary conditions given in Eqs. (27) through (29) are then rewritten as follows:

$$
\begin{aligned}
& \frac{d}{d r} r\left(D_{n} \tilde{\Phi}_{n m}+\frac{2 n(n+1) \tilde{\Phi}_{n m}}{r^{2}}\right)=\frac{d^{2} \tilde{\Phi}_{n m}}{d r^{2}}+\frac{(n-1)(n+2) \tilde{\Phi}_{n m}}{r^{2}} \\
& =D_{n} D_{n} \tilde{\Phi}_{n m}=0, \text { at } r=1 .
\end{aligned}
$$

From the boundary condition for temperature given as Eq. (21) and its regularity at the origin, we can express temperature $\tilde{T}_{n m}$ in terms of spherical Bessel functions, as follows:

$$
\tilde{T}_{n m}=\sum_{j=1}^{\infty} A_{j} \mathcal{J}_{n}\left(\alpha_{n, j} r\right),
$$

where $\alpha_{n, j}$ denotes the $j$-th zero point of the spherical Bessel function of order $n$. Since the spherical Bessel function $\mathcal{J}_{n}(\alpha r)$ satisfies the following ordinary differential equation:

$$
\frac{d^{2} \mathcal{J}_{n}(\alpha r)}{d r^{2}}+\frac{2}{r} \frac{d \mathcal{J}_{n}(\alpha r)}{d r}+\left[\alpha^{2}-\frac{n(n+1)}{r^{2}}\right] \mathcal{J}_{n}(\alpha r)=0
$$

we can obtain the following simple relationship:

$$
D_{n} \mathcal{J}_{n}(\alpha r)=-\alpha^{2} \mathcal{J}_{n}(\alpha r)
$$


In addition, $\mathcal{J}_{n}(\alpha r)$ satisfies the following orthogonal relationship:

$$
\int_{0}^{1} r^{2} \mathcal{J}_{n}\left(\alpha_{n, j} r\right) \mathcal{J}_{n}\left(\alpha_{n, k} r\right) d r=\frac{1}{2}\left[\mathcal{J}_{n+1}\left(\alpha_{n, j}\right)\right]^{2} \delta_{j k} .
$$

From the relationship between temperature and the poloidal potential given as Eq. (32), we obtain the following:

$$
D_{n} D_{n} \tilde{\Phi}_{n m}=\tilde{T}_{n m}=\sum_{j=1}^{\infty} A_{j} \mathcal{J}_{n}\left(\alpha_{n, j} r\right) .
$$

We then search for the poloidal potential in the form of

$$
\tilde{\Phi}_{n m}=\sum_{j} A_{j} \tilde{\Phi}_{n m, j}
$$

Then, we have

$$
D_{n} D_{n} \tilde{\Phi}_{n m, j}=\mathcal{J}_{n}\left(\alpha_{n, j} r\right)
$$

The general solutions of this differential equation, which are regular at the origin $r=0$, are as follows:

$$
\tilde{\Phi}_{n m, j}=\frac{1}{\alpha_{n, j}^{4}} \mathcal{J}_{n}\left(\alpha_{n, j} r\right)+B_{j} r^{n}+C_{j} r^{n+2},
$$

The coefficients $B_{j}$ and $C_{j}$ can be determined from the boundary conditions at $r=1$. From Eq. (34), we have

$$
\begin{aligned}
C_{j} & =\frac{1}{2\left(2 n^{2}+6 n+3\right)}\left[\frac{1}{\alpha_{n, j}}-\frac{2 n(n+2)}{\alpha_{n, j}^{3}}\right] \mathcal{J}_{n}^{\prime}\left(\alpha_{n, j}\right), \\
B_{j} & =-\frac{1}{2(n+1)(n-1)}\left[-\frac{2}{\alpha_{n, j}^{3}} \mathcal{J}_{n}^{\prime}\left(\alpha_{n, j}\right)+2 n(n+2) C_{j}\right] .
\end{aligned}
$$

Substituting $\tilde{\Phi}_{n m}$ with these coefficients into Eq. (31), multiplying it by $r^{2} \mathcal{J}_{n}\left(\alpha_{n, k} r\right)$, and integrating from 0 to 1 , we obtain

$$
n(n+1) \sum_{j} M_{k j} A_{j}+\frac{1}{R} \alpha_{n, k}^{2} \frac{1}{2}\left[\mathcal{J}_{n+1}\left(\alpha_{n, k}\right)\right]^{2} A_{k}=q_{n m, k},
$$

where

$$
\begin{aligned}
& M_{k j}=\int_{0}^{1} r \frac{d T_{0}}{d r}\left[\frac{1}{\alpha_{n, j}^{4}} \mathcal{J}_{n}\left(\alpha_{n, j} r\right)+B_{j} r^{n}+C_{j} r^{n+2}\right] \mathcal{J}_{n}\left(\alpha_{n, k} r\right) d r \\
& q_{n m, k}=\int_{0}^{1} r^{2} \tilde{q}_{J n m}(r) \mathcal{J}_{n}\left(\alpha_{n, k} r\right) d r
\end{aligned}
$$


We can obtain the expression of the steady solution for a given distribution of Joule heating $Q_{J}(r, \theta, \phi)$ by calculating the expansion coefficient $q_{n m, k}$ and solving Eq. (44) to obtain the coefficient $A_{j}$.

The basic temperature profile is proportional to $r^{2}$ when we assume a thermal conductive solution with a constant cooling rate. On the other hand, the radial adiabatic temperature profile $\exp \left(-\alpha\left(g_{0} / a\right) r^{2} / \rho_{0} C_{p}\right)$ is also approximately proportional to $r^{2}$. Then, let us assume the non-dimensionalized basic temperature profile as $d T_{0} / d r \sim r$. Using the recurrence relationship of spherical Bessel functions, Eq. (44) can be simplified as follows:

$$
n(n+1) \sum_{j} M_{k j}^{\prime} A_{j}+\left(\frac{n(n+1)}{\alpha_{n, k}^{4}}+\frac{\alpha_{n, k}^{2}}{R}\right) \frac{1}{2}\left[\mathcal{J}_{n+1}\left(\alpha_{n, k}\right)\right]^{2} A_{k}=q_{n m, k},
$$

where

$$
M_{k j}^{\prime}=\frac{B_{j}}{\alpha_{n, k}} \mathcal{J}_{n+1}\left(\alpha_{n, k}\right)+\left[\frac{2 n+3}{\alpha_{n, k}^{2}} \mathcal{J}_{n+2}\left(\alpha_{n, k}\right)-\frac{1}{\alpha_{n, k}} \mathcal{J}_{n+3}\left(\alpha_{n, k}\right)\right] C_{j} .
$$

Moreover, when the Joule heating distribution is $\tilde{q}_{J n m}=Q_{0} r^{n}, q_{n m, k}$ can be calculated easily.

$$
q_{n m, k}=\frac{Q_{0}}{\alpha_{n, k}} \mathcal{J}_{n+1}\left(\alpha_{n, k}\right) .
$$

3.1. Asymptotic solutions under dominance of the advection of the basic temperature

When the advection term of the basic temperature dominates the diffusion term, i.e., $R \rightarrow \infty$, an expression of the asymptotic solution is easily obtained. In this case, we can neglect the diffusion term in Eq. (44) to obtain

$$
\tilde{\Phi}_{n m}=\frac{r}{n(n+1)\left(d T_{0} / d r\right)} \tilde{q}_{J n m} \quad(n \neq 0) .
$$

As in the above discussion, when we assume the non-dimensional basic temperature profile as $d T_{0} / d r \sim r$, we have

$$
\tilde{\Phi}_{n m}=\frac{1}{n(n+1)} \tilde{q}_{J n m} \quad(n \neq 0) .
$$

The poloidal potential is directly determined by the Joule heating, and their distributions resemble each other. Note that since this velocity distribution 
generally does not satisfy the boundary condition at the ICB, a thin boundary layer would be formed near the ICB to connect the asymptotic solution and the boundary condition. The thickness of this boundary layer, $\delta$, can be roughly estimated from the balance between the advection term of the basic temperature and the diffusion term. From Eq. (26),

$$
\frac{L_{2} \Phi}{r} \frac{d T_{0}}{d r} \sim \frac{1}{R} \nabla^{2} \nabla^{2} \nabla^{2} \Phi
$$

Selecting the length scale as $\delta$, we have

$$
\delta \sim[n(n+1) R]^{-1 / 6} .
$$

\section{Geophysical applications}

In this section, we apply the steady solution obtained in the previous section to the Earth's inner core by considering a specific magnetic field at the ICB and a Joule heating distribution and investigate induced fluid flows.

Important magnetic field components at the ICB for the fluid motion in the Earth's inner core are low-degree toroidal components because they deeply penetrate the inner core. However, the lowest degree components, $n=1$, are not interesting because they produce a uniform Joule heating distribution. The toroidal components of degree greater than one can produce horizontally heterogeneous heating distributions. Then, let us consider a degree-two toroidal component, $\tilde{\mathcal{T}}_{20}$, as the basic magnetic field at the ICB. The magnetic field and Joule heating are expressed as follows:

$$
\begin{aligned}
& \boldsymbol{B}_{0}=B_{0}\left(\frac{r}{a}\right)^{2} \sin 2 \theta \boldsymbol{e}_{\phi}, \\
& Q_{J}=\frac{4 B_{0}^{2}}{\sigma \mu^{2} a^{2}}\left(\frac{r}{a}\right)^{2}\left(3 \cos ^{2} \theta+1\right)=\frac{4 B_{0}^{2}}{\sigma \mu^{2} a^{2}}\left(\frac{r}{a}\right)^{2}\left(2 Y_{2}^{0}+2 Y_{0}^{0}\right),
\end{aligned}
$$

where $\boldsymbol{e}_{\phi}$ is the unit vector in the azimuthal direction. Figure 1 shows the distributions of the magnetic field, associated electric current, and Joule heating. Note that the amplitude of Joule heating is larger in the polar regions than in the equatorial region. The electric current flows axisymmetrically from the equatorial region to the polar regions through the inner part of the core. The 

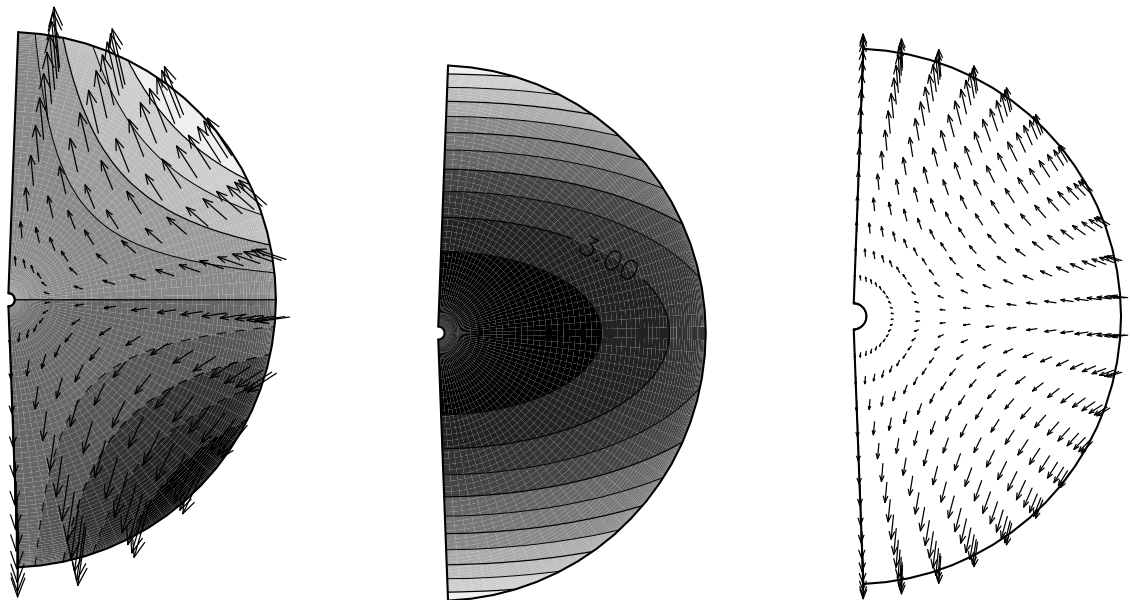

Figure 1: Toroidal $Y_{2}^{0}$ component of the magnetic field (contour lines in the left-hand panel), associated electric current field (arrows in the left-hand panel), Joule heating distribution (center panel), and induced flow fields (right-hand panel) in a meridonal cross section are shown. The amplitude of the basic magnetic field is normalized to unity. The contour intervals are 0.2 and 1.5 in the right-hand and center panels, respectively. In the left-hand panel, the light and dark areas indicate positive and negative values, respectively. In the center panel, the light and dark areas indicate large and small positive values, respectively. The flow field is for the case of $R=10^{7}$.

magnitude of the electric current is greater around the poles than near the equator due to the spherical geometry and the continuity of the current filed. Corresponding to this current field, Joule heating becomes stronger around the polar regions than near the equatorial region.

Since a uniform Joule heating component does not induce fluid flows, it is sufficient to examine the effect of the $Y_{2}^{0}$ component only. Therefore, we select the scaling of Joule heating as $\left|Q_{J}\right|=8 B_{0}^{2} /\left(\sigma \mu^{2} a^{2}\right)$. Since the $Y_{20}$ component of Eq. (53) is proportional to $r^{2}$, we can use Eq. (48), and $q_{n m, k}$ is calculated as $q_{20, k}=\mathcal{J}_{3}\left(\alpha_{2, k}\right) / \alpha_{2, k}$. Then, the coefficients $A_{j}, B_{j}$, and $C_{J}$ in the expression of the steady solution of Eqs. (39) and (40) can be estimated using Eqs. (41), (42), (46), and (47) for a given value of the Rayleigh number $R$. The right panel of Figure 1 presents the flow field distribution for the case in which $R=10^{7}$. The series of spherical Bessel functions are calculated up to the 50th degree. Downwelling is found to occur in the equatorial region, where Joule heating is 
relatively weak, while upwelling occurs in the polar regions, where Joule heating is relatively strong.

Since $R$ is sufficiently large in this case, we can obtain an analytical asymptotic expression of the velocity field. From Eq. (50), the poloidal velocity potential becomes

$$
\Phi=\frac{1}{6} r^{2} Y_{2}^{0}=\frac{1}{12} r^{2}\left(3 \cos ^{2} \theta-1\right)
$$

The velocity components are

$$
\begin{aligned}
& v_{r}=\frac{L_{2} \Phi}{r}=\frac{r}{2}\left(3 \cos ^{2} \theta-1\right), \\
& v_{\theta}=\frac{1}{r} \frac{\partial}{\partial \theta} \frac{\partial(r \Phi)}{\partial r}=-\frac{3 r}{2} \sin \theta \cos \theta \\
& v_{\phi}=0
\end{aligned}
$$

The viscous stress field $\sigma_{i j}^{\prime}$ is

$$
\begin{aligned}
\sigma_{r r}^{\prime} & =\frac{\partial v_{r}}{\partial r}=3 \cos ^{2} \theta-1, \\
\sigma_{\theta \theta}^{\prime} & =\left(\frac{1}{r} \frac{\partial v_{\theta}}{\partial \theta}+\frac{v_{r}}{r}\right)=-3 \cos ^{2} \theta+2, \\
\sigma_{\phi \phi}^{\prime} & =2\left(\frac{1}{r \sin \theta} \frac{\partial v_{\phi}}{\partial \phi}+\frac{v_{r}}{r}+\frac{v_{\theta} \cot \theta}{r}\right)=-1, \\
\sigma_{r \theta}^{\prime} & =\left(\frac{\partial v_{\theta}}{\partial r}+\frac{1}{r} \frac{\partial v_{r}}{\partial \theta}-\frac{v_{\theta}}{r}\right)=-3 \cos \theta \sin \theta=-\frac{3}{2} \sin 2 \theta, \\
\sigma_{\theta \phi}^{\prime} & =\left(\frac{1}{r \sin \theta} \frac{\partial v_{\theta}}{\partial \phi}+\frac{1}{r} \frac{\partial v_{\phi}}{\partial \theta}-\frac{v_{\phi}}{r}\right)=0, \\
\sigma_{\phi r}^{\prime} & =\left(\frac{\partial v_{\phi}}{\partial r}+\frac{1}{r \sin \theta} \frac{\partial v_{r}}{\partial \phi}-\frac{v_{\phi}}{r}\right)=0 .
\end{aligned}
$$

The amplitude of the velocity is approximately unity in a non-dimensional value. Converting the amplitude to the dimensional value, we obtain

$$
|\boldsymbol{v}| \sim \frac{\left|Q_{J}\right|}{\rho C_{p}} \frac{a}{\Delta T} .=\frac{8 B_{0}^{2}}{\rho_{0} C_{p} \sigma \mu^{2} a^{2}} \cdot \frac{a}{\Delta T}=\frac{8 B_{0}^{2}}{\rho_{0} C_{p} \sigma \mu^{2} a \Delta T} .
$$

Note that this estimate of velocity amplitude is independent of the value of viscosity. This is because the velocity field is directly determined by the balance between the advection of basic temperature and the Joule heating in the equation of temperature, rather than by the equation of motion. Applying 
$\mu=4 \pi \times 10^{-7}, \sigma=2 \times 10^{5} \mathrm{Sm}^{-1}, \rho=10^{4} \mathrm{~kg} / \mathrm{m}^{3}, c_{p}=700 \mathrm{~J} / \mathrm{kg} \mathrm{K}$ (Stacey and Davis, 2008), and assuming the magnitude of the toroidal magnetic field at the ICB to be $10^{-2}-10^{-1} \mathrm{~T}$, we have

$$
|\boldsymbol{v}| \sim 1.2 \times 10^{-11}-1.2 \times 10^{-9} \mathrm{~m} / \mathrm{s} .
$$

The amplitude of the strain rate $\dot{\varepsilon}$ becomes

$$
\dot{\varepsilon} \sim \frac{|\boldsymbol{v}|}{a} \sim 1 \times 10^{-17}-1 \times 10^{-15} \mathrm{~s}^{-1} .
$$

When the viscosity of the inner core is assumed to be a moderate value, $\eta=$ $10^{17} \mathrm{~Pa}$ s, the magnitude of the stress field $\sigma_{i j}$ is estimated as follows:

$$
\sigma_{i j} \sim \eta \dot{\varepsilon} \sim 1-10^{2} \mathrm{~Pa} .
$$

Therefore, the predicted magnitude of the stress field is expected to be sufficiently large for deformation of the inside of the inner core.

Figure 2 shows the distribution of the viscous stress field corresponding to the velocity field shown in Figure 1. Here, $\sigma_{\phi \phi}^{\prime}$ is found to be approximately uniform inside the core, whereas other components vary with $\theta$. Note also that a surface boundary layer, which consists of a steep radial gradient of viscous stress, is observed in the distribution of each component of the stress field.

Figure 3 shows the radial distribution of each component of the viscous stress field. The surface boundary layer is clear in the figure. Note that the magnitude of the stress field is smaller in the boundary layer than that in the inner region due to the stress-free boundary condition at the surface.

Figure 4 presents the dependence of the thickness of the surface boundary layer on the Rayleigh number. The boundary layer is found to become thinner as the Rayleigh number increases. The thickness of the boundary layer is proportional to $R^{-1 / 6}$, which is consistent with the theoretical estimate given by Eq. (51) in the previous section. From this relationship, the thickness of the boundary layer is estimated as follows:

$$
\delta \sim a R^{-1 / 6} \sim 6 \times 10^{4} \mathrm{~m}=60 \mathrm{~km} .
$$


(a) $\sigma_{r r}^{\prime}$

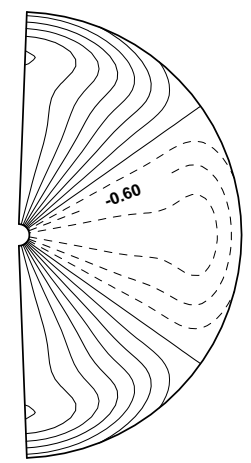

(b) $\sigma_{\theta \theta}^{\prime}$

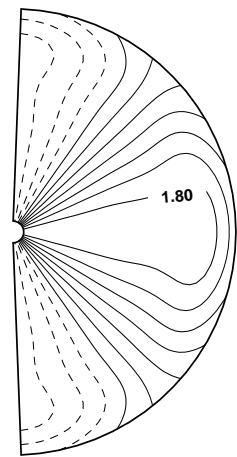

(c) $\sigma_{\phi \phi}^{\prime}$

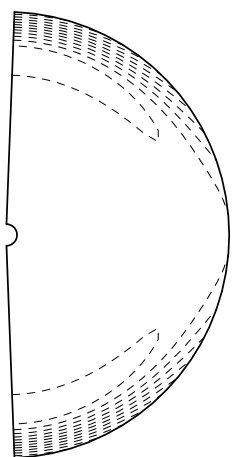

(d) $\sigma_{r \theta}^{\prime}$

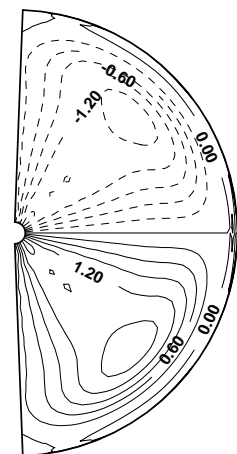

Figure 2: Viscous stress field $\sigma_{i j}^{\prime}$ induced by the toroidal $Y_{2}^{0}$ component of the magnetic field for the case in which $R=10^{7}$. The contour intervals are $0.3,0.6,0.06$, and 0.3 , respectively.

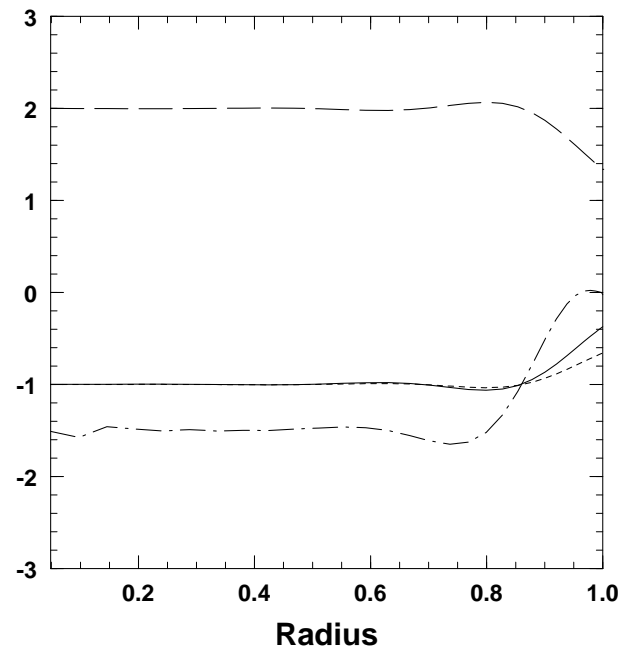

Figure 3: Radial distributions of the viscous stress field induced by the toroidal $Y_{2}^{0}$ component of the magnetic field in the case of $R=10^{7}$. The solid, dashed, dotted, and dash-dotted lines indicate $\sigma_{r r}^{\prime}, \sigma_{\theta \theta}^{\prime}, \sigma_{\phi \phi}^{\prime}$, and $\sigma_{r \theta}^{\prime}$, respectively. Here, $\sigma_{r r}^{\prime}$ and $\sigma_{\theta \theta}^{\prime}$ are shown for the slice at $\theta=\pi / 2$, whereas $\sigma_{\phi \phi}^{\prime}$ and $\sigma_{r \theta}^{\prime}$ are shown for the slice at $\theta=\pi / 4$. 


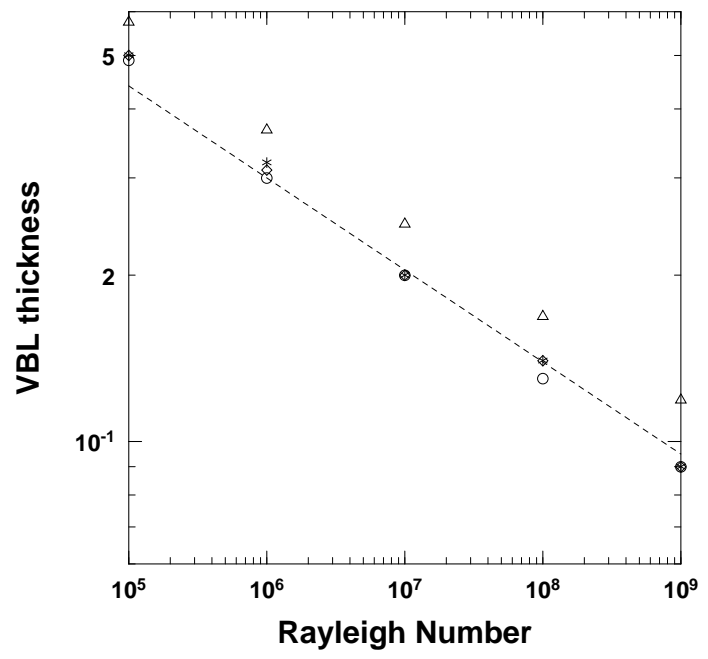

Figure 4: Rayleigh number dependency of the thickness of the surface boundary layer formed by the toroidal $Y_{2}^{0}$ component of the magnetic field. Here, $\star, \circ, \triangle$, and $\diamond$ indicate the thickness of the boundary layer measured from the radial distributions of $\sigma_{r r}^{\prime}, \sigma_{\theta \theta}^{\prime}, \sigma_{\phi \phi}^{\prime}$, and $\sigma_{r \theta}^{\prime}$, respectively. The dotted line indicates $\delta=3 \times R^{-1 / 6}$.

This value is comparable to the depth of the weak anisotropy region observed near the ICB (e.g., Souriau, 2007).

Finally, we estimate the total energy necessary for driving this fluid motion, which is equal to the total Joule heating in the inner core.

$$
\int_{V} Q_{J} d V \sim 1.2 \times 10^{9}-1.2 \times 10^{11} \mathrm{~W} .
$$

This is rather small compared to the estimated energy for maintenance of the dynamo process in the outer core, $\mathrm{O}\left(10^{12}\right) \mathrm{W}$. Therefore, the dynamo process could sufficiently provide the required energy for inducing the fluid flow in the inner core.

\section{Summary and discussion}

We have succeeded in obtaining the analytical solution of the steady fluid flow induced by the horizontal heterogeneous Joule heating generated by the toroidal magnetic field penetrating from the ICB to the inner core. In the 
present study, we consider the thermal effect of the imposed magnetic field, whereas previous studies by Karato (1999) and Buffett and Bloxham (2000) considered the dynamic effects of the imposed magnetic field. A major difference between the thermally induced flow and the dynamically induced flow is that the thermally induced flow does not stop even in a steady state. This is advantageous to the deformation of the inside of the inner core. The fluid motion of this model is driven continuously so that the basic temperature must be advected to balance the Joule heating. Another advantage is that the velocity amplitude is not related to viscosity, the value in the inner core of which is quite ambiguous.

Using the expected values of the physical parameters under the circumstance of the inner core, the order of the estimated magnitude of the stress field is greater than or approximately the same as that of the models considered thus far. Therefore, the fluid flows of this model is expected to be one a candidate for the origin of the seismic anisotropy of the inner core. The anisotropy may be generated mainly by the flows induced by the Joule heating or may be due to the flows driven by the combined effects of the Joule heating and several other factors.

The model also predicts the existence of the surface boundary layer where the stress field is relatively weak. This boundary layer may correspond to the weak seismic anisotropy region that is expected to exist near the ICB. The estimated thickness of the boundary layer of the model is consistent with the depth of the weak seismic anisotropy region.

The model presented in the this study leads to the following suggestions about the dynamic and electromagnetic states in the inner and the outer cores. One suggestion is that the distribution of the seismic anisotropy in the inner core reflects the distribution of the toroidal magnetic field at the ICB. Another is that interactions of the flow and magnetic fields through Joule heating may occur between the inner and outer cores. The mass flux between the inner and outer cores predicted by the model causes the absorption and release of latent heat and light elements at the ICB. For example, the fluid flows induced by the 
toroidal $Y_{2}^{0}$ magnetic field accompany the mass flux from the outer cores to the inner cores around the equatorial region, and from the inner cores to the outer cores around the pole regions (right-hand panel of Figure 1). Thus, latent heat and light elements are released in the equatorial region, and a positive buoyancy source is assigned to the outer core. On the other hand, in the polar regions, latent heat and light elements are absorbed, and a negative buoyancy source is assigned to the outer core. These horizontally heterogeneous buoyancy sources are sufficiently strong compared to the average homogeneous buoyancy source at the ICB, because the velocity amplitude near the ICB is similar to or greater than the average growth rate of the inner core. Therefore, the inner core flows are believed to affect the fluid motions and dynamo action in the outer core through these horizontally heterogeneous buoyancy sources at the ICB and to modify the distribution of the magnetic field in the outer core. Furthermore, based on these suggestions the magnetic field should penetrate to the inner core through the ICB and vary the distribution of the Joule heating and the fluid flows in the inner core. Such an interaction between the magnetic field and the fluid flows between the inner and outer cores may operate through Joule heating. An assessment of the effects of the interaction between the inner and outer cores on the dynamo process in the outer core, such as the stability and maintenance of the generated magnetic field, should be conducted in the future.

\section{Acknowledgements}

The author would like to thank the referees for their useful comments. The products of the Dennou Ruby project (http://www.gfd-dennou.org/library/ruby/) were used to draw the figures.

\section{References}

Bergman, M. I., 1997: Measurements of elastic anisotropy due to solidification texturing and the implications for the Earth's inner core. Nature, 389, 60-63.

Buffett, B. A., 2009: Onset and orientation of convection in the inner core. Geophys. J. Int., 179, 711-719. 
Buffett, B. A., Bloxham, J., 2000: Deformation of Earth's inner core by electromagnetic forces. Geophys. Res. Lett., 27, 4001-4004.

Buffett, B. A., Wenk, H.-R., 2001: Texturing of the Earth's inner core by Maxwell stresses. Nature, 413, 60-63.

Jeanloz, R., 1990: The nature of the Earth's core. Ann. Rev. Earth Planet. Sci., $18,357-386$.

Jeanloz, R., Wenk H.-R., 1988: Convection and anisotropy of the inner core. Geophys. Res. Lett., 15, 72-75.

Karato, S., 1993: Inner core anisotropy due to the magnetic field induced preferred orientation of iron. Science, 262, 1708-1711.

Karato, S. 1999: Seismic anisotropy of the Earth's inner core resulting from flow induced by Maxwell stresses. Nature, 402, 871-873.

Morelli, A., Dziewonski, A. M., Woodhouse J. H., 1986: Anisotropy of the inner core inferred from PKIKP travel times. Geophys. Res. Lett., 13, 1545-1548.

Poupinet, G., Pillet, R., Souriau, A., 1983: Possible heterogeneity in the Earth's core deduced from PKIKP travel times. Nature, 305, 204-206.

Souriau, A., 2007: Deep earth structure - the Earth's cores, In: Schubert, G. (Editor) Treatise on Geophysics, 1, 655-693.

Stacey, F. D., Davis, P. M., 2008: Physics of the Earth. Cambridge University Press, 532pp.

Sumita, I., Bergman, M. I., 2007: Inner-Core Dynamics, In: Schubert, G. (Editor) Treatise on Geophysics, 8, 299-318.

Sumita, I., Yoshida, S., 2003: Thermal interactions between the mantle, outer and inner cores, and the resulting structural evolution of the core. In: Dehant, V., Kenneth, C., Karato, S., Zatman, S. (Editors) Earth's core: dynamics, Structure, Rotation, Geodynamics Series 31, Washington, AGU, 213-231. 
Yoshida, S., Sumita, I., Kumazawa, M., 1996: Growth model of the inner core coupled with outer core dynamics and the resulting elastic anisotropy. J. Geophys. Res., 101, 28085-28103.

Yukutake, T., 1998: Implausibility of convection in the Earth's inner core. Phys. Earth Planet. Inter., 108, 1-13.

\section{A. Non-dimensinalization with thermal diffusion time and velocity}

For the readers who do not become accustomed to the scaling of the variables in the text, we discuss about the derivation of approximate equations (15)-(17) based on the non-dimensinalization with thermal diffusion time and velocity, which is often used for thermal convection problem. Of course, the result is independent of the choice of the scaling units.

The length is scaled with the radius of the sphere $a$. The time scale $\tau$ is selected as $\tau=a^{2} / \kappa$. The velocity scale $V$ is selected as $V=a / \tau=\kappa / a$. The temperature disturbance scale $\Delta T^{\prime}$ is selected as $\left|Q_{J}\right| a^{2} / \rho C_{p} \kappa$ by considering the balance of the Joule heating and the thermal diffusion in the equation of temperature, while the basic temperature is scaled by the typical value of the difference between the basic and the adiabatic temperature, $\Delta T$. The amplitude of the magnetic field disturbance is estimated to be $\left(V a B_{0}\right) / \lambda$ based on the balance between the induction and the magnetic diffusion terms in the induction equation. The pressure term is scaled with $\rho_{0} \nu V / a^{2}$. The non-dimensionalized equations governing the steady state are then obtained as follows:

$$
\begin{aligned}
& \left.\frac{1}{P}(\boldsymbol{v} \cdot \nabla) \boldsymbol{v}+\frac{1}{E} \boldsymbol{e}_{z} \times \boldsymbol{v}=-\nabla p+M^{2}\left(\boldsymbol{J} \times \boldsymbol{B}_{0}+\boldsymbol{J}_{0} \times \boldsymbol{B}\right)+R_{d} T \boldsymbol{r}+\nabla^{\AA} \AA \boldsymbol{v} 1\right) \\
& (\boldsymbol{v} \cdot \nabla) T+\gamma v_{r} \frac{d T_{0}}{d r}=\nabla^{2} T+q_{J} \\
& 0=\nabla \times\left(\boldsymbol{v} \times \boldsymbol{B}_{0}\right)+\nabla^{2} \boldsymbol{B} \\
& \nabla \cdot \boldsymbol{v}=0, \quad \nabla \cdot \boldsymbol{B}=0
\end{aligned}
$$

where $\gamma=\Delta T / \Delta T^{\prime}=\rho C_{p} \kappa \delta T /\left|Q_{J}\right| a^{2}$ is the ratio between the amplitude of basic temperature and temperature disturbance, which is estimated as $\mathrm{O}(10)$ 
by using the typical values of the inner core. $P=\nu / \kappa$ is the Prandtl number, which is as large as $\mathrm{O}\left(10^{18}\right)$. Therefore, the inertial term in the equation of motion can be neglected. Since $E$ and $M$ are estimated in the text as $E \gg 1$ and $M \leq 1$, the Colioris and Lorentz terms also can be neglected. $R_{d}=$ $\alpha g_{0} \Delta T^{\prime} a^{3} / \kappa \nu=\alpha g_{0}\left|Q_{J}\right| a^{5} / \rho C_{p} \kappa^{2} \nu$ is the Rayleigh number. The value of $R_{d}$ is estimated as $R_{d} \sim 8 \times 10^{4}$, which becomes quite large. Then, the main balance in the equation of motion consists of the buoyancy, viscous and pressure gradient terms:

$$
0=-\nabla p+R_{d} T \boldsymbol{r}+\nabla^{2} \boldsymbol{v} .
$$

On the other hand, when the amplitude of each term in the equation of temperature is examined, we have to take care the largeness of $R_{d}$. For example, if we assume that the Joule heating is balanced with the thermal diffusion term, the amplitude of temperature disturbance $T_{d}^{\prime}$ becomes $\mathrm{O}(1)$. Then the velocity amplitude $V_{d}$ becomes $\mathrm{O}\left(R_{d}\right)$ from the equation of motion. This means that the terms of the advection of temperature disturbance and basic temperature become $\mathrm{O}\left(R_{d}\right)$, which dominate the thermal diffusion term.

Moreover, when we assume that the Joule heating is balanced with the advection of temperature disturbance, the relations between the amplitudes of velocity $V_{a}$ and temperature disturbance $T_{a}^{\prime}$ becomes $V_{a} T_{a}^{\prime} \sim \mathrm{O}(1)$. Combined with the force balance $V_{a} \sim R_{d} T_{a}^{\prime}$, the amplitudes of temperature and velocity become $V_{a} \sim \mathrm{O}\left(R_{d}^{1 / 2}\right), T_{a}^{\prime} \sim \mathrm{O}\left(R_{d}^{-1 / 2}\right)$. Since the velocity amplitude becomes large, the advection of basic temperature, which is $\mathrm{O}\left(\gamma R_{d}\right)$, dominates the advection of temperature disturbance.

Therefore, in the equation of temperature, the advection of temperature disturbance and thermal diffusion can be neglected. However, in order to satisfy the boundary condition at the surface of the inner core, we keep the thermal diffusion term,

$$
v_{r} \gamma \frac{d T_{0}}{d r}=\nabla^{2} T+q_{J}
$$




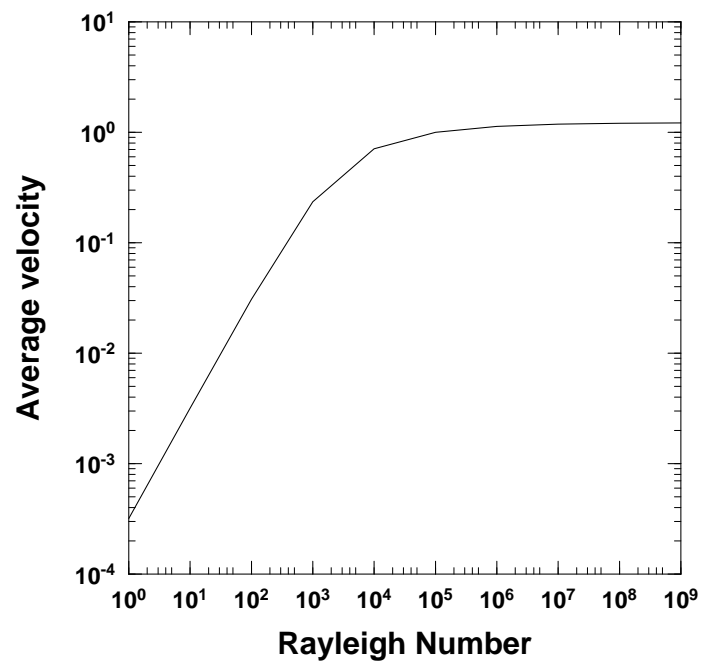

Figure 5: Relationship between the root mean square of the flow field and the Rayleigh number.

\section{B. Rayleigh number dependency of the flow field}

Since we use the expected parameters of the Earth's inner core, only a solution with a large Rayleigh number is examined in Section 4. However, it is worth considering the variation of the flow field using several values of the Rayleigh number in order to clarify the behavior of the model.

Figure 5 shows the relationship between the root mean square of the flow field and the Rayleigh number. The regime changes around $R=10^{4}$. In the regime of $R>10^{4}$, advection of the basic temperature dominates thermal diffusion. This regime is discussed in Section 4. On the other hand, in the regime of $R<10^{4}$, thermal diffusion dominates advection of the basic temperature.

Figure 6 shows the flow field distributions induced by a toroidal $Y_{0}^{2}$ magnetic field for several values of the Rayleigh number. When the Rayleigh number is small and thermal diffusion is dominant, the flow field distribution is deep and the amplitude becomes large around the center of the core. When the Rayleigh number is increased, the depth of the large-amplitude flow field gradually becomes shallow. 
(a) $R=10$

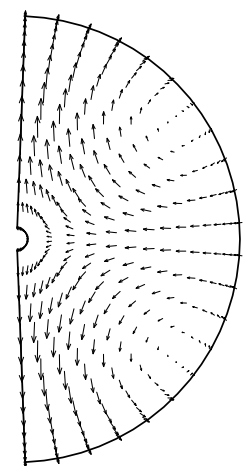

(b) $R=10^{4}$

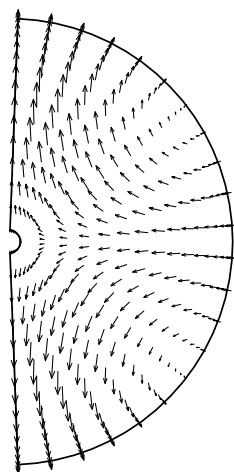

(c) $R=10^{7}$

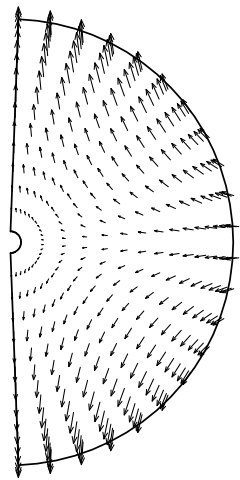

Figure 6: Flow field distributions induced by a toroidal $Y_{0}^{2}$ magnetic field for several values of Rayleigh number in a meridonal cross section.

\section{Asymptotic solution in the case of $R \rightarrow 0$}

Diffusion-dominated solutions might be geophysically insignificant due to the large value of the Rayleigh number of the Earth's inner core. Moreover, the advection of temperature disturbance $(\boldsymbol{v} \cdot \nabla) T$ cannot be neglected in these solutions. Nevertheless, the asymptotic solution in the case of $R \rightarrow 0$ is interesting as an applied mathematics and geophysical fluid dynamics problem. Here, we analytically solve the asymptotic solution of the model for the case in which $R \rightarrow 0$. Neglecting the advection of the basic temperature, Equations (31) and (34) are rewritten as follows:

$$
\begin{aligned}
& -\frac{1}{R} D_{n} D_{n} D_{n} \tilde{\Phi}_{n m}=\tilde{q}_{J n m}, \\
& \frac{d}{d r} r\left(D_{n} \tilde{\Phi}_{n m}+\frac{2 n(n+1) \tilde{\Phi}}{r^{2}}\right)=0, \text { at } r=1, \\
& \frac{d^{2} \tilde{\Phi}_{n m}}{d r^{2}}+\frac{(n-1)(n+2) \tilde{\Phi}_{n m}}{r^{2}}=0, \text { at } r=1, \\
& D_{n} D_{n} \tilde{\Phi}_{n m}=0, \text { at } r=1 .
\end{aligned}
$$

Now, let us assume that the right-hand side of Eq. (C.7) is expressed as a function of $r$ as $\tilde{q}_{J n m}(r)=Q_{0} r^{n_{q}}$. Then, we can obtain the following inhomogeneous 
solution to Eq. (C.7):

$$
\tilde{\Phi}_{s}=D r^{n_{q}+6}
$$

where

$$
D=-\frac{R Q_{0}}{\left(n_{q}-l+6\right)\left(n_{q}+7+l\right)\left(n_{q}-l+4\right)\left(n_{q}+l+5\right)\left(n_{q}-l+2\right)\left(n_{q}+l+3\right)}
$$

Homogeneous solutions of Eq. (C.7), which is regular at $r=0$, are $r^{n}, r^{n+2}$, and $r^{n+4}$. The general solution of Eq. (C.7) is then expressed as follows:

$$
\tilde{\Phi}=A r^{n}+B r^{n+2}+C r^{n+4}+D r^{n_{q}+6},
$$

The coefficients $A, B$, and $C$ are determined by the boundary conditions given in Eqs. (C.8) through (C.10). From Eq. (C.10), we have

$$
C=\frac{Q_{0}}{8(2 l+3)(2 l+5)\left(n_{q}-l+2\right)\left(n_{q}+l+3\right)} .
$$

From Eq. (C.8), we have

$$
\begin{aligned}
& 2 n(n+1)(n-1) A+(n+1)[2(2 n+3)+2 n(n+1)] B \\
& +(n+3)[4(2 n+5)+2 n(n+1)] C \\
& +\left(n_{q}+5\right)\left[\left(n_{q}-n+6\right)\left(n_{q}+n+7\right)+2 n(n+1)\right] D=0 .
\end{aligned}
$$

From Eq. (C.9), we have

$$
\begin{aligned}
& 2(n+1)(n-1) A+2 n(n+2) B a^{n}+[(n+4)(n+3)+(n-1)(n+2)] C \\
& +\left[\left(n_{q}+6\right)\left(n_{q}+5\right)+(n-1)(n+2)\right] D=0 .
\end{aligned}
$$

By eliminating $A$ from Eqs. (C.15) and (C.16), we can obtain the following expression of $B$ :

$$
\begin{aligned}
& B=-\frac{1}{(n+1)[2(2 n+3)+2 n(n+1)]-2 n(n+2)} \times \\
& {[\{(n+3)[4(2 n+5)+2 n(n+1)]-n[(n+4)(n+3)+(n-1)(n+2)] C} \\
& +\left\{\left(n_{q}+5\right)\left[\left(n_{q}-n+6\right)\left(n_{q}+n+7\right)+2 n(n+1)\right]\right. \\
& \left.\left.-n\left[\left(n_{q}+6\right)\left(n_{q}+5\right)+(n-1)(n+2)\right]\right\} D\right]
\end{aligned}
$$


Finally, $A$ is obtained from Eq. (C.13) or Eq. (C.14), as follows:

$$
\begin{aligned}
& A=-\frac{1}{2(n+1)(n-1)} \times\{2 n(n+2) B+[(n+4)(n+3)+(n-1)(n+2)] C \\
& \left.+\left[\left(n_{q}+6\right)\left(n_{q}+5\right)+(n-1)(n+2)\right] D\right\} .
\end{aligned}
$$

